INTRODUCCIÓN

\title{
Economía conductista y derecho penal: nudges, disuasión e interdisciplinariedad
}

\author{
Behavioural Economics and Criminal Law: Nudges, \\ Deterrence, and Interdisciplinarity
}

Fabrizio Esposito ${ }^{* *}$

Recepción y evaluación de propuesta: 06/02/2018

Aceptación: 10/04/2018

Recepción y aceptación final: 16/08/2019

\begin{abstract}
Resumen. Esta introducción tiene dos objetivos. Primero, brinda una breve descripción de los principales temas tratados por las diversas contribuciones. En segundo lugar, coloca las contribuciones en un contexto más amplio. Para este fin, se formulan algunas consideraciones sobre los orígenes de la investigación conductista y su relación con la economía en general y el análisis económico del derecho. Se presta especial atención al concepto de nudge. Luego, se ubica la descripción que Harel y Teichman hacen del análisis económico-conductista del derecho penal en el contexto de la literatura sobre el tema y se señala que Harel y Teichman tienen una descripción muy limitada del campo. La introducción concluye con un llamado metodológico a favor del "minimalismo interdisciplinario", una metodología que ha sido probablemente determinante para el desarrollo de la economía conductista. El minimalismo interdisciplinario puede ayudar a que el análisis económico-conductista del derecho penal prospere y convoque a académicos de diferentes campos para que unan sus fuerzas y reflexionen sobre la división del trabajo que puede mejorar nuestra comprensión del derecho penal y su política criminal.
\end{abstract}

* Traducción del inglés de Fabrizio Esposito y Federico José Arena.

** Ph.D., LL.M. (EUI), Postdoctoral Fellow, Université Catholique de Louvain, Bégica y Extra-mural Fellow, Universidade Nova de Lisboa, Portugal.

Correo electrónico: fabrizio.esposito@eui.eu. 
Fabrizio Esposito

Palabras clave: Análisis económico-conductista, derecho penal, nudge, disuasión, minimalismo interdisciplinario

\begin{abstract}
This introduction to this issue of Discusiones discussing Harel and Teichman's paper has two goals. First, it gives a brief overview of the main themes touch upon by the various contributions. Second, it puts the contributions in a broader context. To this end, some considerations about the origins of behavioural research and its relation with mainstream economics and economic analysis of law are formulated. Special attention is given to the concept of nudge. Then, Harel and Teichman's account of what the behavioural economics analysis of criminal law is is collocated within the literature, to point out that they have a remarkably narrow account of the field. The introduction concludes with a methodological call for 'interdisciplinary minimalism', a methodology which has been arguably important to the establishment of behavioural economics. Interdisciplinary minimalism can help the behavioural economics analysis of criminal law to thrive by calling scholars from different fields to join forces by reflecting on the division of labour that can advance our understanding of criminal law and its policy.
\end{abstract}

Keywords: Behavioural economic analysis, criminal law, nudge, deterrence, interdisciplinary minimalism

\title{
1. Introducción
}

Este número de Discusiones ofrece un debate sofisticado sobre el estado del arte en el ámbito de análisis económico-conductista del derecho penal. Alon Harel y Doron Teichman han investigado sobre estos temas durante muchos años y son expertos destacados en ese campo ${ }^{1}$. Los comentarios de Mag-

1 Ben-Shahar, O. y Harel, A., "Blaming the Victim: Optimal Incentives for Private Precautions against Crime", Journal of Law, Economics, and Organization, 11, 1995, pp. 434-455; Harel, A. y Segal, U., "Criminal Law and Behavioural Law and Economics: Observations on the Neglected Role of Uncertainty in Deterring Crime", American Law and Economics Review, 1, 1999, pp. 276-312; Harel, A. y Klement, A., "The Economics of Stigma: Why More Detection of Crime May Result in Less Stigmatization", Journal of Legal Studies, 36, 2007, pp. 355-377; Harel, A., "Why Only the State May Inflict Criminal Sanctions: The Case Against Privately Inflicted Sanctions", Legal Theory, 14, 2008, pp. 113-133; Harel, A. 
Economía conductista y derecho penal: nudges, disuasión e interdisciplinariedad

dalena Malecka, Anna Richter y Diego Moreno Cruz identifican muchos aspectos del análisis económico-conductista del derecho penal que son de gran interés para los teóricos del derecho y los filósofos interesados en el derecho penal.

En esta introducción tengo la intención de hacer principalmente dos cosas. La primera es proporcionar una breve descripción de los temas tratados por las contribuciones que siguen. La única excepción es la respuesta de Harel y Teichman a Malecka, Richter y Moreno Cruz; dejo que el lector descubra cómo Harel y Teichman han interactuado con sus comentaristas. La segunda es poner las contribuciones en perspectiva, para ayudar al lector a apreciar las complejidades y sutilezas de las cuestiones planteadas por un enfoque económico-conductista del derecho penal. Con este fin, primero realizo algunas consideraciones conceptuales e históricas sobre los orígenes de la investigación económico-conductista y su relación con la economía general y el análisis económico del derecho. Dedico especial atención al concepto de nudge, porque Moreno Cruz lo usa para referirse a todas las propuestas de políticas públicas discutidas por Harel y Teichman. Luego ubico dentro de un contexto más amplio la descripción que Harel y Teichman hacen del análisis económico-conductista del derecho penal y señalo que tienen una concepción muy limitada del campo. Concluiré

y Hylton, K. N. (eds.), Research Handbook on the Economics of Criminal Law, Chelthenam, Edward Elgar, 2012; Harel, A. y Porat, A., "Criminal Responsibility for Unspecified Offences”, Minnesota L. Rev., 94, 2009, pp. 261-310; Alon Harel, “The Duty to Criminalize”, Law and Philosophy, 34, 2015, pp. 1-22; Teichman, D., "The Market for Criminal Justice: Federalism, Crime Control, and Jurisdictional Competition”, Mich. L. Rev., 103 2005, pp. 1831-1876; Teichman, D., "Decentralizing Crime Control: The Political Economy Perspective”, Mich. L. Rev., 104, 2006, pp. 1749-1762; Teichman, D. y Guttel, E., "Criminal Sanctions in The Defense of the Innocent”, Mich. L. Rev., 110, 2012, pp. 597-645; Teichman, D., “The Economics of Crime Control”, en Robinson, P. H., Ferzan, K., Garvey, S. (eds.), Criminal Law Conversations, Oxford, Oxford University Press, 2009, pp. 87-92; Teichman, D., "The Optimism Bias of the Behavioral Analysis of Crime Control”, Ill. L. Rev., 5, 2011, pp. 1697 1712; Teichman, D., Telep, C. W., Weisburd, D., Gill, C. E., Vitter, Z., "Displacement of Crime and Diffusion of Crime Control Benefits in Large-Scale Geographic Areas: A Systematic Review", J. Experimental Criminology, 10, 2014, pp. 515-548; Teichman, D., "Convicting with Reasonable Doubt: An Evidentiary Theory of Criminal Law", Notre Dame L. Rev., 93, 2017, pp. 757-809; Teichman, D. y Zamir, E., The Oxford Handbook of Behavioural Economics and the Law, Oxford, Oxford University Press, 2014; Teichman, D. y Zamir, E., Behavioural Law and Economics, Oxford, Oxford University Press, 2018. 
con un llamado metodológico a favor de una aproximación a la interdisciplinariedad que llamo "minimalismo interdisciplinario". Creo que el minimalismo interdisciplinario ha sido importante para el desarrollo de la economía conductista y que puede ser útil para la prosperidad del análisis económico-conductista del derecho penal. El minimalismo interdisciplinario convoca a los académicos interesados en el derecho penal, independientemente de su campo, a unir fuerzas para reflexionar sobre la división del trabajo que puede mejorar nuestra comprensión del derecho penal y de la política criminal.

Harel y Teichman colocan tres temas en el centro de la agenda para la investigación en el campo del análisis económico-conductista del derecho penal. Primero, la visión del ser humano y del propósito del derecho penal que adopta el análisis económico-conductista se centra en aumentar las conductas en conformidad con el derecho penal -en disuadir el comportamiento criminal al menor costo posible- $y$, en consecuencia, esa visión está en tensión con los enfoques del derecho penal que asumen la visión según la cual los delincuentes merecen el castigo sólo si no pueden justificar su conducta. Se trata de un tema ciertamente abierto a abordajes de teoría y filosofía del derecho. En segundo lugar, ese análisis centrado en los efectos del derecho penal sufre de una significativa indeterminación empírica, dado el estado actual del desarrollo de la economía conductista. En tercer lugar, casi paradójicamente, un derecho penal basado en la disuasión no puede poner el foco exclusivamente en la disuasión cuando los destinatarios tienen fuertes intuiciones a favor de la prioridad de consideraciones retribucionistas. Ignorar estas intuiciones retribucionistas puede reducir drásticamente la inclinación a comportarse en conformidad con el sistema penal, si este está basado en consideraciones de disuasión. Estos dos últimos temas exigen más investigación empírica.

En sus comentarios, Malecka, Richter y Moreno Cruz avanzan sobre estos puntos. No intentaré reconstruir todas sus observaciones, me limitaré a señalar algunas cuestiones relevantes. Malecka defiende una versión aún más fuerte del primer tema planteado por Harel y Teichman: el análisis conductista es incompatible con la concepción "del derecho como una 
Economía conductista y derecho penal: nudges, disuasión e interdisciplinariedad

empresa normativa"' ${ }^{2}$ Sin embargo, su principal objetivo es otro, a saber, señalar que una investigación empírica sobre normas jurídicas bien diseñada presupone una discusión seria acerca de cómo han de modelarse las normas jurídicas mismas, para que sean susceptibles de manipulación en experimentos. Richter explora las relaciones entre la segunda y la tercera cuestiones planteadas por Harel y Teichman, que denomina enfoque de la toma de decisiones y enfoque intuicionista, respectivamente. Este es un análisis particularmente valioso porque, en opinión de Richter, Harel y Teichman consideran que los dos enfoques son independientes y que, en el mejor de los casos, pueden complementarse recíprocamente. En primer lugar, Richter señala que las intuiciones normativas de los destinatarios del derecho penal operan, bajo el enfoque de la toma de decisiones, como una restricción objetiva para lograr la disuasión. Es en el nivel normativo donde Richter identifica las interacciones más delicadas. Comienza por señalar que la disuasión no es "inherente" al análisis económico conductista $^{3}$ y enfatiza que, intuiciones normativas ampliamente compartidas han de tomarse como indicativas de restricciones normativas aplicables al derecho penal, por ejemplo, como limitando su contenido. La coherencia con la moral social puede no ser suficiente para garantizar la justificación del derecho penal. A Richter le preocupan principalmente los puntos de vista minoritarios que no reciben suficiente consideración y también el hecho que la moral social puede ser demasiado indeterminada para proporcionar orientación en relación con aspectos no centrales del derecho penal. Si bien

2 Malecka, M., "Commentarios sobre Harel y Teichman”, en este mismo número. Véase también: Lepenies, R. y Małecka, M., "The Institutional Consequences of Nudging-Nudges, Politics, and the Law", Review of Philosophy and Psychology, 6(3), 2015, pp. 427-437. Para un punto similar, ver: Cserne, P., "Making Sense of Nudge-Scepticism: Three Challenges to EU Law's Learning from Behavioural Sciences", en Alemanno, A. y Sibony, A-L. (eds.), Nudge and the Law, Oxford, Hart Publishing, 2015, pp. 279-299, en pp. 293-297. Para una crítica de este último, véase: Esposito, F., "Nudge and the Law: Book Review", Humana. Mente Journal of Philosophical Studies, 28, 2015, pp. 255-274, en pp. 267-269. Véase también la versión más modesta del argumento en Cserne, P. "Les Nudges Sont-ils des Outils Extra-Juridiques?”, en Brunon-Ernst, A. y Bozzo-Rey, M. (eds.), Nudges et Normativités: Généalogies, Concepts et Applications, Paris, Hermann, 2018, pp. 121-145.

3 Richter, A., "La aplicación del Análisis económico-conductista del derecho a las políticas criminales y sus desafíos", sección 4 , en este mismo volumen. 
la coherencia con la moral social no garantiza la justificación del derecho penal, es de todos modos relevante para identificar cuáles violaciones al derecho penal son más probables y cuál es la mejor manera de aumentar las conductas en conformidad con el derecho penal.

Los comentarios de Moreno Cruz tienen dos objetivos principales: aclarar ciertos aspectos del análisis de Harel y Teichman y presentar como un falso problema la tensión entre el análisis económico-conductista y el modo en que el derecho penal es concebido por la teoría jurídica. Según Moreno Cruz, Harel y Teichman asumen que el análisis económico del derecho tradicional ignora los estados mentales de las personas y explica por qué tal asunción es equivocada. Además, Moreno Cruz identifica una tensión entre el punto de vista según el cual la economía conductista hace foco en la percepción que de la arquitectura de elección tienen los agentes, por un lado, y la inevitabilidad de elegir dentro de esa arquitectura de elección, por otro. Para descartar la primera cuestión planteada por Harel y Teichman, Moreno Cruz defiende un punto de vista normativo pluralista, de acuerdo con el cual la disuasión y el castigo basado en el merecimiento son dos funciones o metas o valores del derecho penal en gran medida consistentes entre sí.

\section{Consideraciones contextuales sobre el análisis económi- co-conductista del derecho}

¿Qué es la economía conductista? Esta pregunta aún no tiene una respuesta convencional y precisa. Sin embargo, hay ciertas consideraciones históricas y conceptuales que determinan características centrales de la economía conductista. La investigación de Daniel Kahneman y Amos Tversky en psicología cognitiva ha sido crucial para el nacimiento de la economía conductista, ya que ha inspirado a una generación de economistas como Richard Thaler, Colin Camerer y George Loewestein. La Universidad de Chicago ha desempeñado un papel importante apoyando al giro conducti- 
Economía conductista y derecho penal: nudges, disuasión e interdisciplinariedad

sta en sus fases iniciales ${ }^{4}$, al igual que la Russell Sage Fundation y el Journal of Economic Perpsectives. Chicago también fue sede de una de las colaboraciones interdisciplinarias más importantes para el éxito global de la economía conductista y para la formulación de políticas públicas, a saber, el encuentro entre Richard Thaler y Cass Sunstein. Junto con Christine Jolls, escribieron en 1998 un artículo famoso y controvertido ${ }^{5}$ titulado "A Behavioural Approach to Law and Economics"6 que fue de suma importancia para que se produzca el giro conductista en el análisis económico del derecho ${ }^{7}$. Y, por supuesto, diez años después, Thaler y Sunstein serían coautores del best seller Nudge.

Conceptualmente, el aspecto clave de la economía conductista es el relajamiento del modelo de elección racional utilizado en la economía. Los agentes tienen una racionalidad limitada y un autointerés limitado, alternan entre procesos mentales rápidos y lentos, y sus decisiones se ven afectadas por sesgos y dependen de la heurística para simplificar el proceso de decisión ${ }^{8}$. Ha habido cambios importantes también a nivel epistemológico y normativo. En particular, la investigación conductista va de la mano con la aceptación, además de la preferencia revelada, de las preferencias explícitas como indicadores de bienestar y con el uso de experimentos de laboratorio para testear el poder predictivo de las teorías económicas. A nivel normativo, en cambio, hay un animado debate sobre cómo es posible

4 Véase el número especial de Journal of Business, 4(2), 1986 y Thaler, R. H., Misbehaving - The Making of Behavioural Economics, New York y London, W. W. Norton \& Company, capítulo 17.

5 Posner, R. A., "Rational Choice, Behavioral Economics, and the Law", Stanford Law Review, 50, 1998, pp. 1551-1576; Kelman, M., "Behavioral Economics as Part of a Rhetorical Duet: A Response to Jolls, Sunstein, and Thaler", Stanford Law Review, 50, 1998, p. 1577-1592.

6 Jolls, C., Sunstein, C. R., Thaler, R. H., "A Behavioural Approach to Law and Economics", Stanford Law Review, 50, 1998, pp. 1471-1550 y Jolls, C., Sunstein, C. R., Thaler, R. H., "Of Theories and Tropes: A Reply to Posner and Kelman", Stanford Law Review, 50, 1998, pp. 1593-1608.

7 En este giro, véase: Esposito, F., "How The Behavioural Turn In Law And Economics Vindicates The New Haven School”, Oeconomia, 7(3), 2017, pp. 375-406.

8 Para una introducción, véase: Thaler, R., Misbehaving, op. cit., y Klaes, M., y Wilkinson, N., An Introduction to Behavioral Economics, 2da ed., London, Palgrave Macmillan, 2012. 
hacer juicios de bienestar, una vez que se acepta que los agentes económicos no son necesariamente los mejores jueces de sus propios intereses. ${ }^{9}$

Teniendo en cuenta este esbozo del contexto, es útil para los propósitos actuales observar que la incorporación de ideas conductistas representa conceptualmente, pero también retóricamente, una enmienda amistosa al análisis económico en general, pero también al derecho. Por ejemplo, Kahneman y Tversky reconocen la importancia para los economistas de la teoría de la utilidad esperada, luego "describen varias clases de problemas de elección en los que las preferencias violan sistemáticamente los axiomas de la teoría de la utilidad esperada" y finalmente "proponen un enfoque alternativo de la elección bajo riesgo"10. La investigación conductista fue presentada desde el principio como importante para la economía general de la época, debido a su capacidad para mejorar la calidad de las predicciones y las explicaciones económicas. Estas importantes características de la investigación conductista, tal como se sostendrá en la última sección de esta introducción, son ilustrativas de una actitud particular hacia la investigación interdisciplinaria que llamo "minimalismo interdisciplinario", que será muy útil para abordar, al menos en parte, las cuestiones planteadas por Harel y Teichman.

\section{Haciendo foco en el nudge}

En su ensayo, Harel y Teichman nunca usan la palabra "nudge"11. Sin embargo, en su comentario, Moreno Cruz se refiere al tema analizado por

9 Véase, por ejemplo, Bernheim, D. y Rangel, A., "Beyond Revealed Preference: Choice-Theoretic Foundations for Behavioral Welfare Economics", The Quarterly Journal of Economics, 124, 2009, pp. 51-104.

${ }^{10}$ Kahneman, D. y Tversky, A., "Prospect theory: an analysis of decision under risk", Econometrica, 47 (2), 1979, p. 263-291. Ver también la column 'Anomalies' que Thaler ha publicado a lo largo de los años en el Journal of Economic Perspectives.

11 [N.T.: "Nudge" es título del libro de Richard Thaler y Cass Sustein. En español el título fue traducido como Un pequeño empujón (Taurus, 2017). Sin embargo, a lo largo de esa traducción se mantiene el término nudge, por lo que también lo usamos en esta edición. Allí, el editor aclara lo siguiente: "Se ha considerado preferible mantener la palabra inglesa 'nudge', que significa literalmente 'empujar suavemente o dar un golpecito en las costillas, 
Economía conductista y derecho penal: nudges, disuasión e interdisciplinariedad

Harel y Teichman como "nudge penal": "un tipo de acción informada comportamentalmente que está encaminada a alterar el comportamiento de las personas al apelar a los estímulos producidos en su mente, y que mantiene la libertad de la gente de obedecer o de encarar" el derecho penal ${ }^{12}$. Es importante reflexionar sobre si la reformulación de Moreno Cruz es feliz o no, porque "nudge" es una palabra de moda hoy en día, y se han expresado preocupaciones sobre el riesgo de que la extensión exagerada del concepto termine por hacerle perder su utilidad. Además, el nudge está fuertemente conectado con el paternalismo libertario, que a su vez tiene un cierto espíritu antirregulador ${ }^{13}$.

Definamos "nudge" como "un efecto de la arquitectura de elección que altera el comportamiento de las personas haciendo uso de uno o más elementos conductistas" -es decir, en virtud de todos aquellos elementos de la toma de decisiones que no son tomados en cuenta por la teoría económica de la elección racional ${ }^{14}$. De acuerdo con esta definición, los nudges pueden ser involuntarios, ineficaces e incluso inexplicables. Pueden ser de interés para quien recibe el $n u d g e$, para quien lo produce, o para terceros o, incluso, para nadie en absoluto. Pueden tener diferentes grados de intrusión fática en la deliberación individual. Por otra parte, y de manera controvertida, los nudges no requieren que usted sea libre de hacer lo que el nudge espera que usted haga.

Sobre este último punto, considérese el ejemplo de la campaña "No te Metas con Texas" (Don't Mess With Texas) que mencionan Thaler y Sunstein en Nudge. Si bien las multas por arrojar basura se habían incrementado, la basura en las carreteras seguía siendo un problema importante. En cambio, una campaña de publicidad con testimonios de famosos

sobre todo con el codo' a fin de 'avisar, recordar o amonestar suavemente a otro'. De un modo más general, en este libro tiene el sentido de estimular, incentivar o encaminar en la toma de decisiones."]

${ }^{12}$ Moreno Cruz, D., "La política criminal del nudge. Algunos desafíos del Análisis conductista a las teorías del Derecho Penal tradicionales", sección 3, en este mismo volumen.

${ }^{13}$ Para una discusión, véase Oliver, A., "Nudging, Shoving, and Budging: Behavioural Economic-Informed Policy”, Public administration, 2015, pp. 1-15.

${ }^{14}$ Véase Esposito, F., "Conceptual Foundations of the Behavioural Analysis of Consumer Law in Europe", en Micklitz, H-W, Sibony, A-L y Esposito, F. (eds.), Research Methods in Consumer Law, Cheltenham, Edward Elgar, 2018, pp. 38-76, en pp. 42-46. 
"amenazando" a quien se metiera con Texas fue muy efectiva para reducir la basura. Para Thaler y Sunstein, esto es un $n u d g e^{15}$. Al mismo tiempo, arrojar basura estaba y sigue estando prohibido en Texas. En consecuencia, ser libre de hacer lo que el nudge espera que uno haga no puede tomarse como una condición necesaria de un nudge. (Sin embargo, la preservación de la elección es, con bastante plausibilidad, una condición necesaria para un nudge coherente con el paternalismo libertario).

Moreno Cruz hace de la intencionalidad un elemento de la definición de nudge. Esto me parece innecesariamente restrictivo; las reglas por defecto a favor de las donaciones de órganos aumentaron el número de donantes de órganos, independientemente de si este fue el efecto deseado. Pero podemos dejar este punto de lado, porque Moreno Cruz plantea un problema crucial en relación nada menos que con el concepto de libertad. Desde su punto de vista, los individuos son libres de elegir si cumplir con el derecho penal o no - y, según Moreno Cruz, esto es clave para hablar de un nudge penal. Sin embargo, no parece totalmente convencido por la posición que ha tomado cuando señala que "una regla penal no es un nudge en un sentido estricto; controlar el comportamiento de las personas por medio de mandatos soportados en amenazas no es una instanciación del tipo de control 'nudging"'16. De hecho, en el caso de las prohibiciones penales, desde el punto de vista del derecho penal, uno no es libre de realizar la acción prohibida - está prohibido, después de todo. Y, sin embargo, uno puede optar por no obedecer el derecho penal. ¿Pero es esto suficiente para considerar a la persona libre? ¿Cuándo, entonces, no somos libres?

Quizás Moreno Cruz busca aplicar el concepto de nudge al derecho penal sin abandonar la idea de que los nudges preservan la libertad de elección. Si uno no es libre de infringir el derecho penal y los nudges preservan la libertad de elección, entonces no existe tal cosa como un nudge penal. Se podría hablar de intervenciones "similares a los nudges" o "cuasi-nudges" o de intervenciones conductistas, que Oliver propone llamar "budge"17. Una de las

15 Thaler, R. H. y Sunstein, C. R., Nudge: Improving Decisions About Health, Wealth, and Happiness, New Haven y London, Yale University Press, 2008, p. 60.

${ }^{16}$ Moreno Cruz, D., op. cit., sección 3.

17 Oliver, "Nudging, Shoving, and Budging", op. cit. 
Economía conductista y derecho penal: nudges, disuasión e interdisciplinariedad

ventajas de hablar de budge penal es que mantiene intacta la conexión entre la preservación de la libertad de elección y los nudges y, al mismo tiempo, se introduce un término para referirse a las intervenciones conductistas en el ámbito penal. Sin embargo, una desventaja es que en el libro que precisamente crea el término icónico "nudge", hay ejemplos de nudges que no preservan la libertad de elección. En consecuencia, se justificaría hablar de nudge penal siempre y cuando el derecho penal, como parte de la arquitectura de elección, tenga el efecto de alterar el comportamiento de las personas haciendo uso de uno o más elementos conductistas.

La cuestión no cuenta actualmente con una solución. Pero al menos espero haber entregado a los lectores algunos instrumentos para navegar a través de los usos, inevitablemente inconsistentes, del término "nudge" que encontrarán en la literatura.

\section{4. ¿De qué trata el análisis económico del derecho penal?}

En sus artículo, Harel y Teichman sostienen que el objetivo central de análisis económico-conductista del derecho penal es cómo mejorar su capacidad disuasoria, es decir, cómo inducir a las personas a cumplir con los preceptos del derecho penal. En esta sección, pretendo ubicar ese punto de vista dentro del contexto más amplio de la literatura sobre el análisis económico del derecho penal ${ }^{18}$. Al hacerlo, mostraré que Harel y Teichman adoptan una visión muy estrecha del análisis conductista del derecho penal. Algunas de las observaciones de Malecka, Richter y Moreno Cruz ilustran la importancia de asumir una perspectiva más amplia.

${ }^{18}$ Introducciones útiles al tema, que sugiero al lector en el orden que sigue, son: Fischer, T., "Economic Analysis of Criminal Law", en Dubber, M. D. y (eds.), The Oxford Handbook of Criminal Law, Oxford, Oxford University Press, 2014, pp. 38-58; Enrilich, I., "Economics of Criminal Law", en Parisi, F. (ed.), The Oxford Handbook of Law and Economics, Vol. 3, Oxford, Oxford University Press, 2017, pp. 295-318; Teichman, D. y Zamir, E., Behavioural Law and Economics, op. cit. 2018; Van Winden, F. y Ash, E., "On the Behavioural Economics of Crime”, Review of Law and Economics, 8(1), 2012, pp. 181-213; Garoupa, N., "Behavioural Economic Analysis of Crime: A Critical Review", European Journal of Law and Economics, 15, 2003, pp. 5-15. 
Como se señaló, Harel y Teichman describen el análisis económico-conductista del derecho penal como centrado en la obtención de la mejor disuasión, es decir, en cómo garantizar, de la manera más eficiente, el cumplimiento del derecho penal. Sin embargo, no explican lo que quieren decir con "disuasión". Cabe destacar a este respecto que se deben distinguir dos enfoques diferentes de la disuasión dentro del análisis económico: el enfoque de la internalización y el enfoque del pleno cumplimiento. El enfoque de la internalización se remonta a la investigación pionera de Becker sobre la economía del comportamiento criminal ${ }^{19}$. Becker adopta una "visión pigouviana"20 de la disuasión, según la cual el derecho penal es un medio para la internalización, por parte de los delincuentes potenciales, de los costos sociales de la delincuencia. Según este punto de vista, la delincuencia debe ser disuadida si y solo si reduce el bienestar social. Cuando los criminales internalizan los costos sociales de sus acciones y prefieren, de todas maneras, actuar, sus acciones aumentan el bienestar social. El enfoque del pleno cumplimiento apunta, en cambio, a evitar todas las violaciones del derecho penal. ${ }^{21}$

Para ilustrar la diferencia, imagine el caso de una computadora personal, que vale \$ 50000 para su dueño. Aprehender y condenar al ladrón tiene, digamos, un costo total de $\$ 40000$ para el sistema legal argentino. La ganancia del ladrón es desconocida. Para el enfoque de la internalización, primero debemos establecer cuáles son los costos sociales del robo, luego establecer una sanción disuasoria si y solo si el robo reducirá el bienestar social. Los costos sociales son el daño directo a la víctima y los costos indirectos de detener y condenar al ladrón: \$ 90000 en total. En consecuencia, si el ladrón está dispuesto a pagar esta suma, significa que, después de haber internalizado los costos sociales, todavía estará mejor y nadie estará peor. En consecuencia, el robo está económicamente justificado de acuerdo

19 Becker, G. S., "Crime and Punishment: An Economic Approach", Journal of Political Economy, 76, 1968, pp. 169-217. Véase también Enrilich, I., "Economics of Criminal Law", op. cit.

20 Para una discusión reciente, vea Masur, J. S. y Posner, E. A., “Toward a Pigouvian State”, U. Pa. L. Rev., 164, 2015, pp. 93-147.

${ }^{21}$ Posner, R. A., "An Economic Theory of the Criminal Law", Columbia Law Review, 85(6), 1985, pp. 1193-1231. Para una discusión crítica, véase Zerbe, R. H., Economic Efficiency in Law and Economics, Chelthenam, Edward Elgar, 2001. 
Economía conductista y derecho penal: nudges, disuasión e interdisciplinariedad

con el enfoque de la internalización puesto que ha aumentado el bienestar social y, por lo tanto, no debería ser castigado. Bajo el enfoque del pleno cumplimiento, en cambio, la sanción debe ser lo suficientemente alta como para garantizar que el ladrón prefiera no violar el derecho penal, incluso cuando la violación implicase una asignación más eficiente de recursos.

Hylton ha analizado la relación entre estos dos enfoques de la disuasión y ha tratado de reconciliarlos teniendo en cuenta la compensación institucional (institutional trade-off) entre una transferencia voluntaria y una involuntaria de recursos ${ }^{22}$. Hylton distingue tres escenarios principales: conductas siempre indeseables; conductas potencialmente indeseables; conductas por fuera del mercado. Es interesante que, en el análisis de Hylton, la meta normativa es la maximización del bienestar social, y lo dejo como una pregunta abierta hasta qué punto el análisis actual es compatible con otros marcos normativos. La intuición principal es que ciertas conductas son siempre socialmente indeseables, mientras que otras solo algunas veces. Al mismo tiempo, en una economía de mercado, los recursos normalmente se transfieren por consenso. La disuasión total parece apropiada para las conductas que son siempre indeseables. Cuando no estamos seguros acerca de la deseabilidad de una conducta, el enfoque de la internalización brinda a los agentes la oportunidad de satisfacer sus preferencias, siempre que estén dispuestos a pagar sus costos sociales. La tercera categoría, la de conductas por fuera del mercado, puede verse como una instancia de la primera. La idea es que, si en relación con un recurso dado hay un mercado que funciona bien, los agentes tienen que satisfacer sus preferencias en ese mercado. Si volvemos al ejemplo de la computadora robada, podríamos ver que la razón por la que tal robo no es deseable está fuertemente relacionada con la constatación de que en Argentina existe un mercado de computadoras portátiles que funciona razonablemente bien. En consecuencia, una transacción voluntaria habría asegurado que el intercambio aumente el bienestar social.

Como se anticipó, un tema que conecta los distintos artículos publicados en este número es la relación que una adecuada comprensión del

${ }^{22}$ Hylton K. N., "The Theory of Penalties and the Economics of Criminal Law", Review of Law and Economics, 1(2), -2005, pp. 175-201. 
derecho penal debe establecer entre la disuasión y el castigo basado en el merecimiento. En este sentido, es notable que el enfoque de la internalización es mucho más contraintuitivo que el enfoque de pleno cumplimiento. El enfoque de la internalización nos da una imagen distorsionada del derecho penal y de las normas jurídicas en general. Por un lado, considérese los casos en que la violación del derecho penal podría ser socialmente deseable. El enfoque de la internalización implica que cuando se viola el derecho penal en este escenario y los delincuentes internalizan los costos sociales, no merecen ninguna estigmatización; en realidad, sus acciones son socialmente deseables. Por otro lado, y más en general, el enfoque de la internalización asume una comprensión de las normas que, de hecho, está en tensión con el papel que la argumentación racional y la justificación tienen en la práctica jurídica. Bajo el enfoque de la internalización, quien a violado la ley penal no tienen la oportunidad de explicar por qué no deberían ser castigados, argumentando, por ejemplo, a favor de una excepción a la prohibición penal de la conducta. Como ya se vio, es el acto de internalizar los costos sociales lo que prueba que la conducta era en realidad deseable. Este enfoque es, por lo tanto, incompatible con la opinión según la cual quien a violado la ley penal merece un estigma social y con la idea de que los procedimientos judiciales dan a los delincuentes la oportunidad de justificar sus conductas.

Podemos ver, en cambio, que respecto a ambos problemas, el enfoque del pleno cumplimiento completo funciona significativamente mejor que el de la internalización. El delito es una conducta socialmente indeseable bajo el enfoque del pleno cumplimiento y, por lo tanto, el delincuente recibe un estigma social. Al mismo tiempo, esta tesis no es en absoluto incompatible con dar al presunto delincuente la oportunidad de justificar su conducta. A la luz de estas consideraciones, las investigaciones que tratan de conciliar las justificaciones del derecho penal basadas en la disuasión con aquellas basadas en el merecimiento deberían tener en cuenta estas notables diferencias entre los enfoques de la internalización y del pleno cumplimiento.

El análisis de Hylton está parcialmente relacionado con una visión más amplia del análisis económico del derecho penal, que se centra en el alcance 
Economía conductista y derecho penal: nudges, disuasión e interdisciplinariedad

que el derecho penal debe tener dentro de un sistema jurídico ${ }^{23}$. No todo lo que recibe una sanción negativa es un delito. Por ejemplo, los impuestos y las indemnizaciones por violaciones al derecho privado también son sanciones negativas, pero no son sanciones penales. Desde esta perspectiva, la distinción entre los enfoques de la internalización y del pleno cumplimiento puede verse como un intento por establecer los límites entre el derecho penal y otras formas de sanción negativa. Ampliar de este modo el análisis es indispensable para abordar la cuestión de los límites del derecho penal y para apreciar la importancia de la disuasión. La cuestión acerca de los límites exige una justificación de por qué una determinada conducta debe ser sancionada por el derecho penal, además o como alternativa al derecho contractual, al derecho fiscal, al derecho administrativo, etc. Sin saber cómo los diferentes enfoques del derecho penal trazan la línea que lo separa de otras ramas del derecho, resulta difícil determinar cuán persuasiva es su concepción de la disuasión en el derecho penal.

Otra línea, menos desarrollada desde mi punto de vista, de la literatura analiza de qué otro modo, además de mediante sanciones negativas, el derecho penal motiva a cumplir con sus preceptos. El derecho en general, y el derecho penal en particular (debido a su estigma social), expresan valores sociales $^{24}$. Saber que el derecho penal prohíbe una acción suele llevar a sus destinatarios a compartir una evaluación negativa de ese curso de acción. Por ejemplo, una prohibición criminal del consumo de alcohol puede llevar a algunos a pensar que es incorrecto consumir alcohol. En otras palabras, el derecho penal puede motivar el cumplimiento moldeando las preferencias de sus destinatarios. Este punto es particularmente relevante en relación con un tema central de esta discusión, a saber, la tensión entre el análisis económico-conductista del derecho penal y la posibilidad de garantizar el cumplimiento, basado en el merecimiento. Harel y Teichman se refieren a esta función expresiva del derecho penal. De hecho, señalan que “(e)1 derecho penal implica a veces un estigma, y está asociado con intuiciones

${ }^{23}$ En este punto, véase Bowles, R., Faure, M., Garoupa, N., "The Scope of Criminal Law and Criminal Sanctions: An Economic View and Policy Implications", Journal of Law and Society, 35(3), 2008, pp. 389-416.

${ }^{24}$ Dau-Schmidt, K. G., "An Economic Analysis of the Criminal Law as a Preference-Shaping Policy", 1, 1990, pp. 1-38. 
morales"25. Incluso más, les preocupa el hecho que las intuiciones normativas, basadas en el merecimiento, de los destinatarios del derecho penal puedan reducir el efecto disuasorio de un sistema de derecho penal basado en la disuasión. Una de las observaciones que hacen para contrarrestar esta preocupación es que las personas podrían de todos modos aceptar un diseño de derecho penal basado en la disuasión pues "aún pueden entender los méritos de la idea si se explica adecuadamente" ${ }^{26}$. Además, podemos conjeturar que, si el derecho penal tiene un efecto expresivo, entonces saber que el derecho penal vigente considera importante la disuasión podría aumentar la aceptación, por parte de sus destinatarios, de consideraciones basadas en la disuasión. Las conexiones apenas identificadas entre la función expresiva del derecho penal y la disuasión bien podrían ser de gran importancia para abordar el conflicto entre los enfoques del derecho penal basados en la disuasión y aquello basados en el merecimiento.

\section{El minimalismo interdisciplinario y el futuro de la dogmática penal}

Me gustaría concluir estos comentarios introductorios sobre el análisis económico-conductista del derecho penal sugiriendo que, el enfoque interdisciplinario de la investigación que llamo "Minimalismo interdisciplinario" ofrece un marco fructífero para fomentar un tipo de colaboración útil, a los fines de abordar los problemas identificados por Harel y Teichman. Según el minimalismo interdisciplinario, las diferentes disciplinas deberían, en la medida de lo posible, poner entre paréntesis sus profundas diferencias e intentar colaborar, incluso bajo incertidumbre, o desacordar acerca de si una pregunta de investigación es interesante ${ }^{27}$.

${ }^{25}$ Harel, A. y Teichman, D., "Análisis conductista del derecho penal y su aplicación: una amistosa revisión crítica”, sección 4.3 , en este mismo volumen.

${ }^{26}$ Harel, A. y Teichman, D., op. cit., sección 5.3.

27 Esposito, F., Law and Economics United in Diversity: Minimalism, Fairness, and Consumer Welfare in EU Antitrust and Consumer Law, Florence, European University Institute, 2018, pp. 4-12; Esposito, F., "On the Fitness Between Law and Economics-Or Sunstein Between Posner and Calabresi”, Global Jurist, 19 (3), 2019, https://doi.org/10.1515/gj-2018-0054. 
Economía conductista y derecho penal: nudges, disuasión e interdisciplinariedad

El minimalismo interdisciplinario es, curiosamente, una instancia más de la estrategia minimalista que, según Sunstein, ha dado forma a su investigación sobre decisión judicial, la democracia deliberativa y el paternalismo libertario. ${ }^{28}$ En relación con la decisión judicial, Sunstein señala que los jueces tienen fuertes razones prima facie morales y pragmáticas para tomar decisiones de alcance más limitado y menos profundas en su justificación ${ }^{29}$. Por un lado, Sunstein señala la importancia de ser respetuosos y tolerantes con las visiones del mundo de los demás. Por otro lado, un enfoque minimalista facilita la colaboración, ya que evita la necesidad de deliberar sobre asuntos controvertidos, siempre que ello sea posible. En relación con el paternalismo libertario, está claro que Sunstein y Thaler han tratado de identificar un conjunto de medidas de gobierno que, en cuanto mantienen la libertad del destinatario para decidir de manera diferente, resultan menos hostiles para los puntos de vistas libertarios. ${ }^{30}$

El minimalismo interdisciplinario, como el minimalismo judicial, enfatiza que el acuerdo para actuar colectivamente, entre agentes que asumen posiciones diferentes, no requiere la eliminación de esas diferencias. Requiere únicamente identificar cursos de acción que sean aceptables para todos los participantes. La identificación de ventajas mutuas debería ser suficiente para asegurar intercambios interdisciplinarios de ideas.

Veamos ahora los problemas planteados por Harel y Teichman, para ver si el minimalismo interdisciplinario puede ser de ayuda para abordarlos. Veamos, primero, el conflicto entre la disuasión y el merecimiento en el diseño del derecho penal. Como se vio en la sección 3, dentro del análisis económico del derecho, hay concepciones muy diferentes de la disuasión

${ }^{28}$ Sunstein, C. R., “On Fallibility: A Reply”, en Morigiwa, Y. y Takikawa, H. (eds.), Judicial Minimalism-For and Against, Stuttgart, Franz Steiner Verlag Sunstein, 2012, pp. 91-96, en p. 94.

29 Sunstein, C. R., Legal Reasoning and Political Conflict, New York, Oxford University Press, 1996; Sunstein, C. R. "From Consumer Sovereignty to Cost-Benefit Analysis: An Incompletely Theorized Agreement", Harvard Journal of Law and Public Policy, 23(1), 1999, pp. 203-210; Sunstein, C. R., "Problems with Minimalism", Stanford Law Review, 58, 2006, pp. 1899-1918; Sunstein, C. R., “Beyond Judicial Minimalism”, en Morigiwa, Y. y Takikawa, H. (eds.), Judicial Minimalism, op. cit., pp. 13-33.

${ }^{30}$ Sunstein, C. R. y Thaler, R. H., "Libertarian Paternalism", The American Economic Review, 93(2), 2003, pp. 175-179. 
y, además, es posible dar espacio a la capacidad motivadora de las elecciones valorativas expresadas por el derecho penal. Además, como señala Moreno Cruz, entre los teóricos y los filósofos del derecho existe una gran variedad de opiniones sobre el papel de la disuasión en el derecho penal. Una reflexión cuidadosa acerca de hasta qué punto estas diferentes perspectivas en el análisis económico y la teoría del derecho encajan entre sí, sería un paso importante para comprender hasta qué punto las opiniones son realmente incompatibles.

Nótese que el positivismo jurídico no juega un papel importante en el análisis de Harel y Teichman. Sin embargo, el positivismo jurídico contribuiría a este intercambio interdisciplinario, arrojando luz sobre la medida en que las diferentes perspectivas encajan con la práctica jurídica real. Especialmente para aquellos economistas que desean centrarse en los análisis empíricos, no parece problemático aceptar las elecciones de valor expresadas por el derecho como relevantes para determinar el diseño del derecho penal. Al mismo tiempo, un análisis jurídico positivista también sería útil para educar a los destinatarios acerca de la importancia de la disuasión. Por ejemplo, Harel y Teichman sostienen que el aumento de las sanciones para los reincidentes es incompatible con un derecho penal basado en el merecimiento. En la medida en que esto sea correcto, el hecho de que el derecho penal castigue más severamente a los reincidentes puede ser útil para desafiar la opinión popular según la cual la disuasión tiene un papel menor dentro del derecho penal.

Este punto nos lleva a la importancia que la colaboración interdisciplinaria tiene para completar nuestra comprensión de cómo de facto funciona el derecho penal, lo que, a su vez, es crucial para recopilar los datos que, según Harel y Teichman, se necesitan para mejorar la base empírica del análisis económico-conductista. Como ellos observan, no hay mucha información empírica sobre muchos temas relevantes, y aún más importante, parte de la información proviene de estudios que no tenían por objeto el derecho penal. Además, tal como Malecka señala en sus comentarios, el modelado de las normas jurídicas en contextos experimentales es un tema delicado y todavía poco investigado. Los juristas definitivamente pueden contribuir al diseño de la investigación empírica necesaria para ofrecer resultados confiables. En consecuencia, parece que hay amplio espacio para 
la colaboración. Los juristas están interesados en conocer los efectos del derecho penal, y una mejor modelización de las normas jurídicas sería de gran utilidad para la investigación empírica.

Por supuesto, existen barreras institucionales a esta colaboración interdisciplinaria. Barreras tales como, por ejemplo, la falta de oportunidades para interactuar, las diferencias en la trayectoria profesional y las dificultades para encontrar revistas que estén interesadas en este tipo de colaboración interdisciplinaria. Sin embargo, a la luz de la discusión anterior, parece claro que tal colaboración es deseable y, por lo tanto, debería ser fomentada. Esperamos que este número especial motive a algunos lectores a explorar diferentes maneras de contribuir a un estudio interdisciplinario del derecho penal.

\section{Bibliografía}

Becker, G. S., "Crime and Punishment: An Economic Approach", Journal of Political Economy, 76, 1968, pp. 169-217.

Ben-Shahar, O. y Harel, A. "Blaming the Victim: Optimal Incentives for Private Precautions against Crime”, Journal of Law, Economics, and Organization, 11, 1995, pp. 434-455.

Bernheim, D. y Rangel, A., "Beyond Revealed Preference: Choice-Theoretic Foundations for Behavioral Welfare Economics", The Quarterly Journal of Economics, 124, 2009, pp. 51-104.

Bowles, R., Faure, M., Garoupa, N., "The Scope of Criminal Law and Criminal Sanctions: An Economic View and Policy Implications", Journal of Law and Society, 35(3), 2008, pp. 389-416.

Cserne, P., "Making Sense of Nudge-Scepticism: Three Challenges to EU Law's Learning from Behavioural Sciences", en Alemanno, A. y Sibony, A-L. (eds.), Nudge and the Law, Oxford, Hart Publishing, 2015, pp. 279-299.

Cserne, P. “Les Nudges Sont-ils des Outils Extra-Juridiques?”, en BrunonErnst, A. y Bozzo-Rey, M. (eds.), Nudges et Normativités: Généalogies, Concepts et Applications, Paris, Hermann, 2018, pp. 121-145. 
Fabrizio Esposito

Dau-Schmidt, K. G., "An Economic Analysis of the Criminal Law as a Preference-Shaping Policy", 1, 1990, pp. 1-38.

Enrilich, I., "Economics of Criminal Law", en Parisi, F. (ed.), The Oxford Handbook of Law and Economics, Vol. 3, Oxford, Oxford University Press, 2017, pp. 295-318.

Esposito, F., "Nudge and the Law: Book Review", Humana.Mente Journal of Philosophical Studies, 28, 2015, pp. 255-274.

Esposito, F., "How The Behavioural Turn In Law And Economics Vindicates The New Haven School”, Oeconomia, 7(3), 2017, pp. 375-406.

Esposito, F., Law and Economics United in Diversity: Minimalism, Fairness, and Consumer Welfare in EU Antitrust and Consumer Law, Florence, European University Institute, 2018

Esposito, F., "Conceptual Foundations of the Behavioural Analysis of Consumer Law in Europe", en Micklitz, H-W, Sibony, A-L, Esposito, F. (eds.), Research Methods in Consumer Law, Cheltenham, Edward Elgar, 2018, pp. 38-76, a pp. 42-46.

Esposito, F., "On the Fitness Between Law and Economics-Or Sunstein Between Posner and Calabresi”, Global Jurist, 19 (3), 2019, https://doi. org/10.1515/gj-2018-0054.

Fischer, T., "Economic Analysis of Criminal Law", en Dubber, M. D. y (eds.), The Oxford Handbook of Criminal Law, Oxford, Oxford University Press, 2014, pp. 38-58.

Garoupa, N., "Behavioural Economic Analysis of Crime: A Critical Review", European Journal of Law and Economics, 15, 2003, pp. 5-15. Harel, A., "Why Only the State May Inflict Criminal Sanctions: The Case Against Privately Inflicted Sanctions", Legal Theory, 14, 2008, pp. 113133.

Harel, A., "The Duty to Criminalize", Law and Philosophy, 34, 2015, pp. $1-22$.

Harel, A. y Klement, A., "The Economics of Stigma: Why More Detection of Crime May Result in Less Stigmatization", Journal of Legal Studies, 36, 2007, pp. 355-377.

Harel, A. y Segal, U., "Criminal Law and Behavioural Law and Economics: Observations on the Neglected Role of Uncertainty in Deterring Crime", American Law and Economics Review, 1, 1999, pp. 276-312. 
Economía conductista y derecho penal: nudges, disuasión e interdisciplinariedad

Harel, A. y Hylton, K. N. (eds.), Research Handbook on the Economics of Criminal Law, Chelthenam, Edward Elgar, 2012.

Harel, A. y Porat, A., "Criminal Responsibility for Unspecified Offences", Minnesota L. Rev., 94, 2009, pp. 261-310.

Harel, A. y Teichman, D., "Análisis Conductista del Derecho Penal y su Aplicación: Una Amistosa Revisión Crítica”, Discusiones, 22 (2), 2018.

Harel, A. y Teichman, D., "Enfrentar las Críticas", Discusiones, 22 (2), 2018. Hylton K. N., "The Theory of Penalties and the Economics of Criminal Law", Review of Law and Economics, 1(2), -2005, pp. 175-201.

Jolls, C., Sunstein, C. R., Thaler, R. H., "A Behavioural Approach to Law and Economics", Stanford Law Review, 50, 1998, pp. 1471-1550.

Jolls, C., Sunstein, C. R., Thaler, R. H., “Of Theories and Tropes: A Reply to Posner and Kelman", Stanford Law Review, 50, 1998, pp. 1593-1608.

Kahneman, D. y Tversky, A., "Prospect theory: an analysis of decision under risk", Econometrica, 47 (2), 1979, pp. 263-291.

Kelman, M., "Behavioral Economics as Part of a Rhetorical Duet: A Response to Jolls, Sunstein, and Thaler", Stanford Law Review, 50, 1998, pp. 1577-1592.

Klaes, M., y Wilkinson, N., An Introduction to Behavioral Economics, 2nd edn, London, Palgrave Macmillan, 2012.

Lepenies, R. y Małecka, M., “The Institutional Consequences of NudgingNudges, Politics, and the Law", Review of Philosophy and Psychology, 6(3), 2015, pp. 427-437.

Malecka, M., "Commentarios Sobre Harel y Teichman", Discusiones, 22 (2), 2018.

Masur, J. S. y Posner, E. A., “Toward a Pigouvian State”, U. Pa. L. Rev., 164, 2015, pp. 93-147.

Moreno Cruz, D., "La Política Criminal del Nudge. Algunos desafíos del Análisis Comportamentalista a las teorías del Derecho Penal tradicionales", Discusiones, 22 (2), 2018.

Oliver, A., "Nudging, Shoving, and Budging: Behavioural EconomicInformed Policy", Public administration, 2015, pp. 1-15.

Posner, R. A., "An Economic Theory of the Criminal Law", Columbia Law Review, 85(6), 1985, pp. 1193-1231. 
Fabrizio Esposito

Posner, R. A., "Rational Choice, Behavioral Economics, and the Law", Stanford Law Review, 50, 1998, pp. 1551-1576.

Richter, A., "La Aplicación del Análisis Económico Conductista del Derecho a las Políticas Criminales y sus Desafíos”, Discusiones, 22 (2), 2018.

Sunstein, C. R., Legal Reasoning and Political Conflict, New York, Oxford University Press, 1996.

Sunstein, C. R. "From Consumer Sovereignty to Cost-Benefit Analysis: An Incompletely Theorized Agreement", Harvard Journal of Law and Public Policy, 23(1), 1999, pp. 203-210.

Sunstein, C. R., "Problems with Minimalism", Stanford Law Review, 58, 2006, pp. 1899-1918.

Sunstein, C. R., “Beyond Judicial Minimalism”, en Morigiwa, Y. y Takikawa, H. (eds.), Judicial Minimalism-For and Against, Stuttgart, Franz Steiner Verlag Sunstein, 2012, pp. 13-33.

Sunstein, C. R., "On Fallibility: A Reply”, en Morigiwa, Y. y Takikawa, H. (eds.), Judicial Minimalism-For and Against, Stuttgart, Franz Steiner Verlag Sunstein, 2012, pp. 91-96.

Sunstein, C. R. y Thaler, R. H., "Libertarian Paternalism", The American Economic Review, 93(2), 2003, pp. 175-179.

Teichman, D., "The Market for Criminal Justice: Federalism, Crime Control, and Jurisdictional Competition", Mich. L. Rev., 103 2005, pp. 1831-1876.

Teichman, D., "Decentralizing Crime Control: The Political Economy Perspective”, Mich. L. Rev., 104, 2006, pp. 1749-1762.

Teichman, D. y Guttel, E., "Criminal Sanctions in The Defense of the Innocent”, Mich. L. Rev., 110, 2012, pp. 597-645.

Teichman, D., “The Economics of Crime Control”, en Robinson, P. H., Ferzan, K., Garvey, S. (eds.), Criminal Law Conversations, Oxford, Oxford University Press, 2009, pp. 87-92.

Teichman, D., "The Optimism Bias of the Behavioral Analysis of Crime Control", Ill. L. Rev., 5, 2011, pp. 1697-1712.

Teichman, D., "Convicting with Reasonable Doubt: An Evidentiary Theory of Criminal Law", Notre Dame L. Rev., 93, 2017, pp. 757-809.

Teichman, D., Telep, C. W., Weisburd, D., Gill, C. E., Vitter, Z., "Displacement of Crime and Diffusion of Crime Control Benefits in 
Economía conductista y derecho penal: nudges, disuasión e interdisciplinariedad

Large-Scale Geographic Areas: A Systematic Review", J. Experimental Criminology, 10, 2014, pp. 515-548.

Teichman, D. y Zamir, E., The Oxford Handbook of Behavioural Economics and the Law, Oxford, Oxford University Press, 2014.

Teichman, D. y Zamir, E., Behavioural Law and Economics, Oxford, Oxford University Press, 2018.

Thaler, R. H. y Sunstein, C. R., Nudge: Improving Decisions About Health, Wealth, and Happiness, New Haven y London, Yale University Press, 2008.

Thaler, R. H., Misbehaving - The Making of Behavioural Economics, New York y London, W. W. Norton \& Company.

Van Winden, F. y Ash, E., "On the Behavioural Economics of Crime", Review of Law and Economics, 8(1), 2012, pp. 181-213.

Zerbe, R. H., Economic Efficiency in Law and Economics, Chelthenam, Edward Elgar, 2001. 